\title{
Designing Visual Interfaces to Support Voice Input
}

\author{
The case of a TV application to request help in daily life tasks
}

\author{
Rita Santos \\ Águeda School of \\ Technology and \\ Management,DigiMedia \\ University of Aveiro \\ Aveiro, Portugal \\ rita.santos@ua.pt
}

\author{
Joana Beja \\ Communication and Art \\ Department, DigiMedia \\ University of Aveiro \\ Aveiro, Portugal \\ joanabeja@ua.pt
}

\author{
Mário Rodrigues \\ Águeda School of \\ Technology and \\ Management, Institute \\ of Electronics and \\ Telematics Engineering \\ of Aveiro \\ University of Aveiro \\ Aveiro, Portugal \\ mjfr@ua.pt
}

\author{
Ciro Martins \\ Águeda School of \\ Technology and \\ Management, Institute \\ of Electronics and \\ Telematics Engineering \\ of Aveiro \\ University of Aveiro \\ Aveiro, Portugal \\ ciro.martins@ua.pt
}

\begin{abstract}
In the last years, voice has been used as an interaction mode in several devices such as smart speakers, mobile devices or television (TV). However, there seems to be a lack of practical recommendations about how visual interfaces can be designed to support the use of voice input in TV applications in certain tasks in a way to take advantage of the full potential of this interaction mode. This paper aims to present the design strategies adopted in a TV application targeting older adults which allows them to request help from a group of volunteers in daily life tasks and where voice is used in certain tasks such as to make the request instead of using the TV remote control. These strategies are discussed around three dimensions considered especially important when using voicebased input: information for voice usage, keeping the user informed and prompts to clarify system's doubts. The paper also presents the results of usability tests with potential users, in order to identify usability problems regarding the use of voice-based input to make the request and to gather information about how improve the usability of this feature. Study results indicate positive attitudes towards the use of voice input method and that older adults considered the use of voice easier than if they had to use a TV remote control to make a request. In a context where voice interaction appears as relevant for older adults, this study can
\end{abstract}

\footnotetext{
Permission to make digital or hard copies of all or part of this work for personal or classroom use is granted without fee provided that copies are not made or distributed for profit or commercial advantage and that copies bear this notice and the full citation on the first page. Copyrights for components of this work owned by others than ACM must be honored. Abstracting with credit is permitted. To copy otherwise, or republish, to post on servers or to redistribute to lists, requires prior specific permission and/or a fee. Request permissions from Permissions@acm.org.

Interacción 2019, June 25-28, 2019, Donostia, Gipuzkoa, Spain

(C) 2019 Association for Computing Machinery.

ACM ISBN 978-1-4503-7176-6/19/06 ..\$15.00

https://doi.org/10.1145/3335595.3335637
}

contribute to more informed design strategies in multimodal interaction targeting this group.

\section{CCS CONCEPTS}

- Human-centered computing User interface design • Humancentered computing Natural language interfaces • Humancentered computing Text input

\section{KEYWORDS}

Interactive television, multimodal interfaces, design recommendations, voice-based input, usability, older adults.

\section{ACM Reference format:}

R. Santos, J. Beja, M. Rodrigues and C. Martins. 2019. Designing Visual Interfaces to Support Voice Input: The case of a TV application to request help in daily life tasks. In XX International Conference of Human Computer Interaction (Interacción'19), June 25-28, 2019, Donostia, Gipuzkoa, Spain. ACM, New York, USA, 8 pages. ISBN 978-1-4503-7176-6/19/06.

https://doi.org/10.1145/3335595.3335637

\section{Introduction}

According to the World Health Organization, the number of people aged 60 years or older will rise from 900 million to 2 billion between 2015 and 2050 (moving from 12\% to 22\% of the total global population) and population ageing is happening more quickly than in the past [1], [2]. The importance of promoting active ageing is widely recognized, which involves helping to create conditions for older adults to maintain their individuality and contribute to the society. The potential of digital media to promote active ageing is widely recognized, although previous research studies suggested that the elderly, in general, use less Information and Communication Technologies (ICT) and have more difficulties in using them than younger adults although it is 
moreover important not to consider this group as a homogeneous one [1], [3]. The complexity of the user interfaces and interaction modalities are some of the possible explanation factors of the difficulties in use [4], [5]. In this way, increasing the number and type of interaction modalities available could contribute to adapting ICT to suit the constraints and needs of specific users such as the elderly. Providing control mechanisms that are flexible, intuitive and robust, by means of multimodal interfaces, seems to be a relevant and helpful solution [6], [7]. In this context, voice has emerged as particularly interesting in diverse types of applications targeting older adults [8] and this is also valid in the context of television (TV) applications [7], although there are some evidences that older adults are not yet willing to abdicate from the TV remote control [9].

As in the mobile context, apps are gradually more present in the TV, provided by broadcaster's operators, Smart TV manufacturers or streaming devices as Chromecast. Being recognized that older adults are the group that watch more TV and that are less attached to other technologies such as smartphones or computers, this group should not be ignored as potential users of TV applications [8]. In this context, the idea of developing a TV application that aims to address needs which are common in this age group, such as provide a ride to the hairdresser, help carrying goods from a store or help keeping the garden in good order, but which, for some reason, often need some external support, seem to be valuable as one can help to raise their confidence and self-esteem. This is particularly valuable considering that the design of services and applications for older adults has not always being concerned about specific characteristics and needs of that type of users [4], reducing its usefulness, accessibility and efficiency.

The VoluntAge4Seniors is a service that has, as a main driver, a TV application which intends to connect older adults and a network of volunteers, providing an easy and quick way for older adults to ask for help in daily life tasks. The final aim is to provide more independence for elderly people, giving them a quick way to access a supervised network of volunteers who will see and respond to the requests on a Web platform (not described in the context of this work). To overcome difficulties related to the use of TV remote control in the context of information provision, it is proposed that some actions in the TV application, such as make the request and leave a comment about the volunteer, can be made by voice. As stated before, the use of voice as a text input method might represent a valuable option, helping to build more accessible applications. However, in contexts where this interaction mode coexists with others, it is important to design solutions where this integration is natural and fluid to the user. Some authors had suggested a set of guidelines and recommendations, but they seem to appear in a more theoretical way. Regarding this, it would be important that research further discuss possible design options to concretize these recommendations.

It is under this context that this paper presents the set of strategies adopted in VoluntAge4Seniors TV application regarding the design of the visual interfaces to support voice input and the results of the validation of these strategies with a group of potential users. To achieve this, previous research works about projects and applications that use voice as an input method with a special focus on the TV context and older adults were analysed, as well as research works that point some guidelines or recommendations considered important to take into account when designing interfaces that use voice as an input method.

After the Introduction in Section 1, the present paper analyses the related work in Section 2, presenting research projects targeting older adults that use voice as a means of interaction and research works that propose guidelines that can be relevant to the design of visual interfaces to support voice-based input. After that review, the strategies adopted in the design of visual interfaces to support voice input of VoluntAge4Seniors TV application are presented in Section 3. The results of usability tests with a group of potential users are presented in Section 4, as well as a set of design recommendations that emerged from this study which can be useful to another research works. The Section 5 present the conclusions and future work.

\section{Related work}

One of the main recognized advantages of voice is its potential to be a way to text input [12]. Voice has already been used as an input method in several devices such as voice assistants like Siri or Google Assistant (for mobile phones), services which convert audio messages to text messages or mobile applications that provide voice input to facilitate the interaction, as DOM Assistant to order Domino's Pizza or Spotify Voice to interact with Spotify features. In the last years, cloud-based speech-to-text solutions such as the ones provided by Microsoft or Google presented a great evolution and efforts have been made to allow the use of a more natural language. Despite the significant improvement that occurred in automatic speech recognition (ASR) systems, accuracy is still an issue [13] and certain solutions still present restrictions such as lack of support for variants of a certain language.

In the TV context, the use of voice is mainly aimed to navigate and control the TV based on a relatively restricted set of voice commands (such as "change to channel 4" or "search Woody Allen") that are spoken out loud by the users after activating the voice button on the TV remote control with microphone incorporated or using a smart-assistant connect to the TV. Besides that, due to some technological constraints, as the requirement of having a Smart TV, a voice-enabled set-top box, or a TV remote control with microphone incorporated, the integration of voice features to interact with TV is still low. Nevertheless, there is increasing interest in more and complex voice features [14], evidenced in the appearance of products such as Dragon TV or Amazon Fire TV, predicting that soon TVs will be in general prepared to allow voice interaction, namely in TV applications as is already happening with mobile apps or smart-speakers. 
Despite these advances, TV remote control is still a widely used device, even being recognized that the interaction with the TV remote control still holds some obstacles, especially in certain contexts [15], such as in conditions associated with aging as vision loss or motor difficulties. In this way, voice can be an alternative to the use of TV remote control especially in what concerns to input great amounts of information, which represents a very timeconsuming process.

\subsection{Use of Voice by older adults}

The use of voice as a way of interaction has been suggested as having benefits [16]-[20], especially for improving end-user accessibility, which is particularly relevant to the older adults. In [21] the authors performed a study with older adults by carrying out a user evaluation of a voice-only system interaction, the Siri application, where no touch or keyboard/ mouse input was possible. By pressing the Siri activation button on the smartphone, the participants were able to directly access the smartphone services via voice without accessing the smartphone display. Even though the participants appreciated the simplicity of that means of interaction, and showed a high acceptance towards it, the system performance, i.e. the voice interaction accuracy, was reported as a problem. In addition, they identified usability issues. Initiating the voice interaction by pressing the Siri activation button sometimes led to the problem that the participants forgot to push it and started speaking without the system listening to them, or they pressed it too shortly not activating the Siri application. In [18], a research study which highlighted some of the challenges involved in designing voice technologies for elderly people, the mechanism for the voice interaction, a button to open/close the microphone channel, revealed a similar issue. Most participants either forgot to press the button or did not release it before talking to the system, suggesting the need for clear visual or auditory feedback to indicate whether voice interaction is on or off.

In the context of TV, due to the high use of television by older adults, there are also several research projects that have been using voice interaction to deliver applications related with personal healthcare or to reduce social isolation and loneliness. One aspect that is highlighted in most of the research work analysed is that users do not discard the use of a TV remote control and prefer to use speech input as a complement to it [11], [17]

In [11] the authors describe how an adapted graphical user interface together with multimodal interaction (TV remote control and voice interaction) was created to integrate diversified interactive systems such as home automation or social interaction with friends. The results from tests with users showed that, despite the participants finding the overall system to be both satisfactory and efficient to use, some concerns were raised regarding the accuracy of the voice interaction. Also [20] conducted an experiment with a group of elderly using voice interaction to control an interactive TV-based application, comparing it with other interaction modalities, namely non-verbal gestures. Voice interaction was positively perceived by the elderly, but only if other traditional control devices were not available. Although users were also able to use gestures, they preferred voice interaction, considering the use of non-verbal gestures awkward.

\subsection{Design Recommendations for Visual Interfaces with Voice Input}

During the study, we also aimed to identify related works approaching recommendations on how user interfaces should be designed to support multimodality, specially voice-based input. Given the aim of the study, we looked mainly for recommendations that could influence the design of the visual component of the interfaces.

Based on presented by [10], the following topics highlight a set of relevant guidelines that can be relevant to this context:

1. Avoid unnecessarily presenting information in two different modalities. Such redundancy can increase cognitive load at the cost of learning the material;

2. Maximize the advantages of each modality to reduce user's memory load in certain tasks and situations;

3. Match output to acceptable user input style;

4. Use multimodal cues to improve collaborative speech;

5. Ensure system output modalities are well synchronized temporally;

6. Ensure the current system interaction state is shared across modalities and that appropriate information is displayed;

7. Adapt the quantity and method of information presentation to both the user and display device;

8. Presentation and prompts should share common features as much as possible and should refer to a common task including using the same terminology across modalities.

It was also aimed to identify design recommendations and best practices from research works approaching the TV context. In [17], the authors indicate positive results in what concerns to speech input, considering it as more effective than remote control, and also reflect about the benefits of combining the flexibility of voice interaction with the strength of visual representation, referring that brings several advantages over speech-only interfaces. Among these, the authors pointed the possibility to present feedback graphically (e.g. to confirm a request), the amount of information that is possible to show and the viewer's attention that can be oriented to specific information. Based on the results of the study, the authors identified some initial insights to be considered to design interfaces for interactive TV multimodal systems, among which are:

1. The remote control should be kept, even when speech is used for interacting with the TV;

2. Graphically inform users about the system capabilities regarding functionality and data. 
In this study is also highlighted that the feedback is particular important in spoken language interfaces and pointed that one of the problems of designing multimodal interfaces is the difficulty to inform the user when each modality is available or appropriated to use [17].

As a result of GUIDE Project, which main goal was to provide guidelines to support interaction with TV Applications for the elderly [7], the authors presented a set of topics to improve usability of TV Applications considering the age-inherent limitations of older adults. These guidelines were also emphasized later with the development and evaluation process of "You, Me \& TV" - a multimodal TV Application for older adults to use Facebook features on television [22]. The authors derived important topics about the multimodal systems, such as:

1. Provide modality help to explain the use of different available devices and training steps for each modality of interaction;

2. Consider the context of use to improve recognition, and aspects related to elderly speech (e.g. pauses) to prevent ASR errors;

3. Present recognized commands or multi-modal feedback to enable error recovery.

After the revision undertaken in 2.1 and 2.2 we can conclude that although several studies had develop visual applications targeting older adults that use voice input and that some authors derived important guidelines to design effective multimodal interfaces that optimize human performance and make system interaction as intuitive as possible, there seems to be little focus about considerations and options taken in terms of the visual interface design, which could inform other studies around this topic.

The following section presents the decisions taken during the design of the visual interfaces of VoluntAge4Seniors TV Application, an application targeting older adults that uses voice as an input method.

\section{Design of the Visual Interfaces of VoluntAge4Seniors TV App to Support Voice Input}

VoluntAge4Seniors is a TV App which allows older adults to request help from a group of volunteers in daily life tasks, such as a ride to the supermarket or simple household maintenance as changing a lamp. To facilitate this task, voice is used as the inputmethod to provide information about the request, through a phone call.

Some studies with older adults has showed some advantages of input information through a phone call, such as the ease of use resulting from the familiarity with the device [23], [24]. We also consider that this approach has the advantage of being adequate for scenarios where voice-enabled set-top boxes or TVs do not exist and that eliminate the need of pressing a button to record the request and releasing it in the end when the user finishes speaking, as happens in the scenario of using a TV remote control with microphone incorporated.

The VoluntAge4Seniors TV App also allows to track the status of the requests (in analysis, awaiting a volunteer, awaiting for the older adult confirmation) and, after the request is made, to evaluate the help that was provided by the volunteer, where voice input can be also used to leave a comment about the service provided.

\subsection{An overview of VoluntAge4Seniors TV App Interface}

After choosing who is going to use the VoluntAge4Seniors TV App, the main screen "Requests" (Pedidos) is presented to the user (Fig. 1), with a fixed left side bar menu that is common to all screens and contain four options: Requests (Pedidos), Personal data (Dados), About (Sobre) and Exit (Sair). The Request screen intends to be a dashboard in which the users can access to the set of principal actions available to the user such as make a new request, confirm the acceptance of help from a volunteer, cancel a request or evaluate volunteers.

\section{Figure 1: Requests Dashboard}

In what concerns navigation, it is possible to navigate using directional arrows and "OK" keys from the TV remote control, as suggested by recommendations [7] [25]. These navigation possibilities are presented to users in one of the first screens of the App.

Regarding the process of making a new request, the user has, in the TV application, to: (1) to ask for receive a phone call; (2) answer to the automatic call in his/her phone and hearing a message asking to leave the request after the beep sound; (3) make the request, hang up the call and; (4) on the TV App, confirm the intention of sending the request to a network of a volunteers. The scheme in Fig. 2 aims to synthetize this process, which involves the Twilio service responsible to make and record the phone call, and the ASR system of Google API that converts the speech into text. 


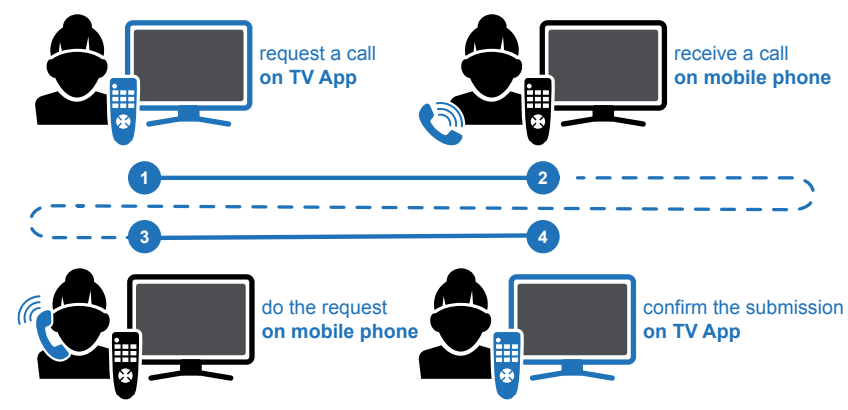

Figure 2: Multimodality when making a request process

This voice input process is also used in the evaluation feature, in which the older adult can give feedback about a request that has already occurred associating a verbal comment. For both scenarios in which voice is used, the text result of the ASR conversion is not immediately displayed to the user, because previous usability tests of the application and other studies as showed that users feel uncomfortable when the conversion is not accurate. For that reason, the result of audio-to-text conversion is only presented on the volunteers' web platform along with the audio file. Here, the volunteer has the opportunity of correcting some mistakes that can come from the transcription of the ASR and send the right information to the TV application.

\subsection{Results of the visual interfaces design}

The following topics describe the visual interfaces design strategies adopted to help the user to utilise the voice input in VoluntAge4Seniors TV App. These strategies are organized around three topics: information for voice usage (intend to prepare the users to make their requests, reducing the doubts and, consequentially, the errors); keep them informed about the process and; clarify some doubts that can appear after making the request. It is worth to mention that these strategies took into consideration the research described in Section 2, Nielsen's heuristics [26], since they are largely applicable for the design of different types of user interfaces [27], as well as the results of intermediary evaluations with potential users and experts that were taken during the design process [28].

3.2.1 Information for voice usage. The strategies discussed below intends to prepare the users to utilize the voice modality, ensuring that they are informed about the necessary steps to make a request, including the use of voice as input method.

\subsubsection{Instructions}

The first screen presented after the user chooses to make a request explain how this process will happened using voice, by showing the necessary steps that the user will have to follow (Fig. 3). These steps include the following information: the need of having the mobile phone near the user, follow the
INTERACCIÓN 2019, June, 2019, Donostia, Gipuzkoa, Spain

steps that will appear on the screen (described in 3.2.1.2), answer the call and say the request, end the call after saying it, and confirm the data about the request and the intention of submit it.

Figure 3: Instructions - "what you need to make a request"

\subsubsection{Script and examples}

Even before the user ask to receive the phone call, and after choosing one of the available categories of the request, the interface presents a script about the ideal construction of a request. It defines a structure composed by "I need" (Preciso de) + "what" ( o quê) + "when" (quando) (Fig. 4). In addition, an example of the script is provided according to the chosen category.

\section{Figure 4: Script suggesting the request structure}

Although the system is prepared to understand other request structures, the script solution not only intends to work as a mechanism to reduce errors [7], as well as aims to create more confidence for users when making their requests. Previous tests without script interface showed some insecurity about how to make a request, which resulted in longer sentences with unnecessary details about the needed support.

The request script is also presented when the older adult initiates the phone call, allowing the user to have the continuous support of the suggested structure while making the request. 
3.2.2 Keep the user informed. This topic presents the visual strategies concerning the need of keeping the user informed about what happens during the phone call, as suggested by the "Visibility of systems status" Nielsen's heuristic [26].

After choosing to initiate the phone call, feedback is also provided on the screen with graphical highlights, as suggested by [7], showing a text label with the sequential steps that the process follows: "calling", "ongoing call" (while the user ...), and "processing your request" (when the user...) (Fig. 5). Messages of "busy" and "rejected call" are also provided, and in these cases the application return to the previous screen. It is also provided an icon near the text label that emphasizes the status message. This communication clearly represents the sharing of information across modalities as suggested by [7].

Also, in what concerns to feedback, it is important to refer that, after the user answers the call, there is an automatic message that informs the user to start his/her request after a beep sound.

Figure 5: Feedback provided during the phone call

3.2.3 Prompts to clarify systems' doubts. This topic presents the visual strategies applied when it is necessary to clarify system's doubts resulting from the request made by voice.

After processing the request, the system analyses if there is a margin to misinterpretations of the sentences, such as dubious or undefined hours, and even requests with low confidence by the ASR system, usually caused by silence or completely nonsensical sentences. These situations lead to different system messages, triggering prompt windows that are displayed with direct questions about the dubious part, presenting multiple choice buttons to facilitate the answer. As shown in the example of Figure 6, the system intends to confirm if the suggested hour is before or after noon. Moreover, the prompt window also provides the possibility to repeat the request call at this point, providing flexibility in cases when, for example, the user is not satisfied with his/her voice request. Another example of system's prompt is when the users do not specify any hour or any date for their request. In this case, the system presents three different options: let the volunteer set the hour, define it manually using the remote control, or repeat the phone call. Finally, bearing in mind the speech characteristics of older adults as, for example, the pauses, we considered that it was important to consider a system's prompt when the ASR result confidence was low, stating that an error has occurred when processing the request and suggesting two options: repeat the call or cancel the request. In this way, we support users to recover from an ASR error, following what some guidelines and heuristics suggest [7], [26].

Figure 6: Doubts' clarification pop-up windows (dubious hours example)

\section{Evaluation and Consequent Recommendations}

After implementing the strategies referred in the previous section, a set of usability tests were conducted with 12 potential users, in which eight of them were female and the other four were male. The average age of the group were 70 years, and although 10 participants had access to a paid television service in their homes, only one of these assumed to be able to schedule recordings of TV shows and none of the participants assumed to be familiar with controlling the emission using the rewind, play or pause features. A half of the participants indicated to use Internet and 7 have a smartphone. The tests were conducted in laboratory settings in order to understand their experience using the system and the participants were invited to explore the following tasks: open the Application, make a request, accept a volunteer, evaluate a volunteer, and close the Application. Behind these tasks, there were a set of defined objectives to understand their difficulties on those specific moments. After the execution of each task, the participants classified that experience using the Single Ease Question (SEQ), which is a seven-point scale to answer the question "Overall, how difficult or easy was the task to complete?", in which 1 represents "very difficult" and 7 "very easy" [29].

Based on the goals of the presented paper, we present here the usability evaluation results of the "make a request" task, since it included all the strategies applied to support voice input previously described. In this task, the participant experienced all the steps of making a new request, from the moment that initiates a new request using the remote control on the TV Application, to the moment that the participant receives a phone call to express their request, confirming it on the TV App at the end. 
All participants completed the task, understanding the multimodality of interaction with the different steps. Despite this, 7 participants did not follow the script structure strictly when they expressed the request during the phone call, expecting a more conversational model. However, they also expressed valid structures to the requests. Also, two users did not hang up the call after saying the request, waiting for some feedback on the other side. Five participants formulated their requests following perfectly the script, and the average result of SEQ in "make a request" task was five points.

In the post-test interview, applied with the aim of gathering feedback regarding the usefulness and the suitability of voice-based input to make a request, 10 participants recognized the utility of the Application, referring that it could be especially useful for persons who live alone. Also, participants' opinion about the suitability of making a request using voice was unanimous, claiming that it easier to speak than use remote control to write the request. Despite this, two participants said that a conversational model would be better, with feedback on the other side.

Based on the results of the study, it is presented here a set of design recommendations that can complement others suggested in previous research works, including:

1. the presentation of a preparation screen with all the steps that the user must follow to use a new modality;

2. a script suggesting a structure that should be followed accompanied with examples, in order to increase the success of the recognition by the ASR system;

3. a screen showing the status of the interaction, meeting the guideline suggested by [7] about feedback with a graphical highlight and;

4. use of prompts that appears when the system detects that some information is missing or when there are some doubts.

\section{Conclusions}

The combination of multiple modes of interaction demands some design principles and options that are different from the ones considered in the design of more traditional visual interfaces. From the research literature review undertaken, it was possible to conclude that some principles and recommendations have been suggested by other authors, although they tend to be more conceptual and generic. This paper aimed to contribute to a more informed design of TV interfaces that use voice as an alternative to text entry, approaching concrete solutions that can be useful to address a set of challenges imposed by the use of voice in combination with visual interfaces and use of a TV remote control. The results of the evaluation presented in this paper revealed that the visual representations to support voice input and the way of inputting voice (using a phone call) were seen as valuable and provided an enjoyable user experience. In a general way, they supported the use of voice in combination with the screens and use of TV remote control, reducing the users' doubts and, consequentially, the errors, and helping them when some problem occurs. We consider that not showing the result of the ASR system soon after the request is made revealed to be a good option since the accuracy of the ASR system sometimes revealed errors which can be problematic specially in a context where people are not used to utilize voice as an input style.

Despite the target group being older adults, we consider that the user interface would also be suited to other user groups that prefer/need simpler interfaces and could benefit from having voice input methods. Further work thus intends to conduct a pilot test with a group of other publics, in order to better validate the receptiveness of the design options implemented to support the voice input. Also, it could be interesting to adapt the visual representations implemented throughout this study to the use of other voice input devices has a TV remote control with microphone integrated or a smart-speaker connected to the TV in order to understand their receptiveness.

\section{ACKNOWLEDGMENTS}

The research leading to this work received funding from AlticeLabs@UA. The authors would like to thank the support provided by the National Association of Gerontologists in Portugal.

\section{REFERENCES}

[1] K. G. Vroman, S. Arthanat, and C. Lysack, “'Who over 65 is online?' Older adults' dispositions toward information communication technology," Comput. Human Behav., vol. 43, pp. 156-166, 2015 WHO, "Ageing and health." [Online]. Available: https://www.who.int/news-room/fact-sheets/detail/ageing-and-health L. C. van Boekel, S. T. Peek, and K. G. Luijkx, "Diversity in Older Adults' Use of the Internet: Identifying Subgroups Through Latent Class Analysis," J. Med.Internet Res., vol. 19, no. 5, p. e180, 2017.

[4] S. Silva, "Developing technologies for the elderly: To whom are we really developing?," in 201537 th Annual International Conference of the IEEE Engineering in Medicine and Biology Society (EMBC), 2015, pp. 8030-8033.

[5] A. Teixeira et al., "Speech-centric Multimodal Interaction for Easy-toaccess Online Services - A Personal Life Assistant for the Elderly," Procedia Comput. Sci., vol. 27, pp. 389-397, 2014.

[6] M. Alaoui and M. Lewkowicz, "Practical issues related to the implication of elderlies in the design process - The case of a Living Lab approach for designing and evaluating social TV services," IRBM, vol. 36, no. 5, pp. 259-265, 2015.

[7] J. Coelho, T. Guerreiro, and C. Duarte, "Designing TV Interaction for the Elderly -- A Case Study of the Design for All Approach," in A Multimodal End-2-End Approach to Accessible Computing, P. Biswas, C. Duarte, P. Langdon, L. Almeida, and C. Jung, Eds. London: Springer London, 2013, pp. 49-69.

[8] C. Dodd, R. Athauda, and M. T. P Adam, "Designing User Interfaces for the Elderly: A Systematic Literature Review," 2017, no. December, pp. $1-11$.

[9] J. Coelho, F. Rito, and C. Duarte, "'You, me \& TV' - Fighting social isolation of older adults with Facebook, TV and multimodality," Int. J. Hum. Comput. Stud., vol. 98, pp. 38-50, 2017.

[10] L. M. Reeves et al., "Guidelines for multimodal user interface design," Commun. ACM, vol. 47, no. 1, pp. 57-59, 2004.

[11] G. Epelde et al., "Providing universally accessible interactive services through TV sets: implementation and validation with elderly users," Multimed. Tools Appl., vol. 67, no. 2, pp. 497-528, Nov. 2013.

[12] A. L. Smith and B. S. Chaparro, "Smartphone Text Input Method Performance, Usability, and Preference with Younger and Older Adults," Hum. Factors, vol. 57, no. 6, pp. 1015-1028, 2015.

[13] V. Këpuska and G. Bohouta, "Comparing Speech Recognition Systems (Microsoft API, Google API And CMU Sphinx)," Int. J. Eng. Res. Appl. Vol 07, Iss 03, Pp 20-24 VO - 07, no. 03, p. 20, 2017. 
[14] J. Abreu, P. Beça, R. Santos, B. Cardoso, S. Fernandes, and A. Rodrigues, "Voice Interaction on TV: Analysis of natural language interaction models," Proc. XIX Int. Conf. Hum. Comput. Interact., p. 8:1-$8: 8,2018$.

[15] V. S. Ribeiro, A. I. Martins, A. Queirós, A. G. Silva, and N. P. Rocha, "Usability Evaluation of a Health Care Application Based on IPTV," Procedia Comput. Sci., vol. 64, pp. 635-642, 2015.

[16] A. Ibrahim and P. Johansson, "Multimodal Dialogue Systems: A Case Study for Interactive TV," in Universal Access Theoretical Perspectives, Practice, and Experience, 2003, pp. 209-218.

[17] A. Berglund and P. Johansson, "Using speech and dialogue for interactive TV navigation," Univers. Access Inf. Soc., vol. 3, no. 3, pp. 224-238, 2004.

[18] S. Schlögl, M. Garschall, and M. Tscheligi, "Designing Natural Language User Interfaces with Elderly Users," in Workshop on Designing Speech and Language Interactions at the ACM SIGCHI Conference on Human Factors in Computing Systems, 2010.

[19] H. Delgado, A. Rodriguez-Alsina, A. Gurguí, E. Martí, J. Serrano, and J. Carrabina, "Enhancing Accessibility through Speech Technologies on AAL Telemedicine Services for iTV," in Ambient Intelligence, 2011, pp. 300-308.

[20] V. Bures, "Interactive digital television and voice interaction: Experimental evaluation and subjective perception by elderly," Elektron. ir Elektrotechnika, vol. 122, no. 6, pp. 87-90, Jun. 2012.

[21] L. Wulf, M. Garschall, J. Himmelsbach, and M. Tscheligi, "Hands Free Care Free: Elderly People Taking Advantage of Speech-only Interaction," in Proceedings of the 8th Nordic Conference on Human-Computer Interaction: Fun, Fast, Foundational, 2014, pp. 203-206.

[22] J. Coelho, F. Rito, and C. Duarte, "'You, me \& TV' - Fighting social isolation of older adults with Facebook, TV and multimodality," Int. J. Hum. Comput. Stud., vol. 98, pp. 38-50, 2017.

[23] R. Ball and J. P. Hourcade, "Rethinking reading for age from paper and computers," Int. J. Hum. Comput. Interact., vol. 27, no. 11, pp. 10661082, 2011

[24] R. Brewer, R. C. Garcia, A. M. Piper, D. Gergle, and T. Schwaba, "Exploring Traditional Phones as an E-Mail Interface for Older Adults," ACM Trans. Access. Comput., vol. 8, no. 2, pp. 1-20, 2016.

[25] SAPO, "Regras de Usabilidade para Navegação." [Online]. Available: https://ux.sapo.pt/usabilidade/tv/navegacao-3/.

[26] J. Nielsen, "10 Usability Heuristics for User Interface Design," 1994. .

[27] E. Wong, "Heuristic Evaluation: How to Conduct a Heuristic Evaluation," Interaction Design Foundation. [Online]. Available: https://www.interaction-design.org/literature/article/heuristic-evaluationhow-to-conduct-a-heuristic-evaluation

[28] T. Ribeiro, R. Santos, J. Moura, A. I. Martins, and H. Caravau, "Application for Older Adults to Ask for Help from Volunteers Through Television: Design and Evaluation of a High Visual-Fidelity Prototype,' Commun. Comput. Inf. Sci., vol. 813, pp. 32-43, 2018.

[29] P. Laubheimer, "Beyond the NPS: Measuring Perceived Usability with the SUS, NASA-TLX, and the Single Ease Question After Tasks and Usability Tests," 2018. [Online]. Available:

https://www.nngroup.com/articles/measuring-perceived-usability/. 